\title{
Commissioning of the SLAC Linac Coherent Light Source II electron source
}

\author{
F. Zhou $\odot,{ }^{1, *}$ C. Adolphsen, ${ }^{1}$ A. Benwell, ${ }^{1}$ G. Brown, ${ }^{1}$ D. H. Dowell, ${ }^{1}$ M. Dunning, ${ }^{1}$ \\ S. Gilevich, ${ }^{1}$ K. Grouev, ${ }^{1}$ G. Huang, ${ }^{2}$ B. Jacobson, ${ }^{1}$ X. H. Liu, ${ }^{1}$ A. Miahnahri, ${ }^{1}$ \\ F. Sannibale, ${ }^{2}$ J. Schmerge, ${ }^{1}$ and T. Vecchione \\ ${ }^{1}$ SLAC National Accelerator laboratory, Menlo Park, California 94025, USA \\ ${ }^{2}$ LBNL National Laboratory, Berkeley, California, USA
}

(Received 28 April 2021; accepted 8 July 2021; published 23 July 2021)

\begin{abstract}
For the Linac Coherent Light Source II (LCLS-II) project at SLAC, a $1.3 \mathrm{GHz}$ superconducting rf (SRF) linac is being constructed that will generate $4 \mathrm{GeV}$ electron bunches at a high repetition rate to drive $\mathrm{x}$-ray free electron lasers. The LCLS-II electron source, which comprises the first three meters of the electron injector, includes two normal-conducting, continuous-wave rf cavities: a one-cell, $185.7 \mathrm{MHz}$ gun and a two-cell, 1.3 GHz buncher. It also includes a gun load-lock system that allows photocathodes to be changed under vacuum. The components in this beam-line section were designed and built by Lawrence Berkeley National Laboratory based on experience from their advanced photoinjector experiment program. In combination with the SLAC UV laser system, the electron source is designed to produce beam rates up to $1 \mathrm{MHz}$ with average currents up to $30 \mu \mathrm{A}$ initially. The source was installed in mid-2018, well in advance of the SRF linac, which is now nearing completion. The source was commissioned over a two-year period, and this paper presents results including electron beam and dark current characterization.
\end{abstract}

DOI: 10.1103/PhysRevAccelBeams.24.073401

\section{INTRODUCTION}

X-ray free electron lasers [1-4] have proven to be a revolutionary tool for photon science studies. The SLAC National Accelerator Laboratory (SLAC) Linac Coherent Light Source II (LCLS-II) project [5], which is nearing completion, represents a major advance in that it will provide up to $1 \mathrm{MHz}$ bunch repetition rates, a substantial increase from the $120 \mathrm{~Hz}$ rate in the existing LCLS facility. This higher rate will allow experiments that require a large number of photon pulse interactions to resolve molecular structure or ultrafast molecular phenomena. The photons will be generated in two x-ray undulator lines from electrons provided by a $4 \mathrm{GeV}$ linac that uses $1.3 \mathrm{GHz}$ superconducting radio frequency (SRF) technology. The electron injector for this linac is designed to generate $100 \mathrm{MeV}, 100 \mathrm{pC}, 12 \mathrm{~A}$ peak current electron bunches with a normalized emittance of about $0.4 \mu \mathrm{m}$ [6-7]. The electron source, which comprises the first three meters of the injector, was designed and built by Lawrence Berkeley National Laboratory (LBNL) following their decade-long advanced photoinjector experiment (APEX) program to develop a $186 \mathrm{MHz}$ normal-conducting (NC), cw electron

\footnotetext{
*zhoufeng@slac.stanford.edu

Published by the American Physical Society under the terms of the Creative Commons Attribution 4.0 International license. Further distribution of this work must maintain attribution to the author(s) and the published article's title, journal citation, and DOI.
}

gun [8-10]. For the LCLS-II project, modifications to the gun design were made based on lessons learned and specific LCLS-II requirements. The electron source parameters are summarized in Table I.

Figure 1 shows a schematic layout of the electron source, which includes a $186 \mathrm{MHz}$ quarter-wave-resonator-style rf gun, a $1.3 \mathrm{GHz}$ two-cell NC cavity for compressing the bunch length, two solenoidal magnets for beam focusing and emittance compensation, and five pairs of dipole magnets for orbit corrections. Beam diagnostics include two beam position monitors (BPMs), a yttrium-aluminumgarnet (YAG) screen, a current monitor (toroid), and a Faraday cup (FC) for measuring the bunch charge and dark current. The FC has since been removed, and the first LCLS-II cryomodule has been attached to the end cap assembly shown in the figure. A photocathode load-lock system that is based on the Instituto Nazionale di Fisica Nucleare and Laboratory for Accelerators and Applied Superconductivity (LASA) design [11] extends upstream

TABLE I. LCLS-II electron source parameters.

\begin{tabular}{lc}
\hline \hline Parameters & Nominal \\
\hline Gun energy $(\mathrm{keV})$ & 750 \\
Gun cathode gradient $(\mathrm{MV} / \mathrm{m})$ & 19.5 \\
Cathode $\mathrm{QE}$ & $>0.5 \%$ \\
Laser energy $(\mu \mathrm{J})$ on the cathode & 0.3 \\
Maximum bunch repetition rate $(\mathrm{MHz})$ & 0.93 \\
Nominal bunch charge $(\mathrm{pC})$ & 100 \\
Initial beam current $(\mu \mathrm{A})$ & 30 \\
\hline \hline
\end{tabular}




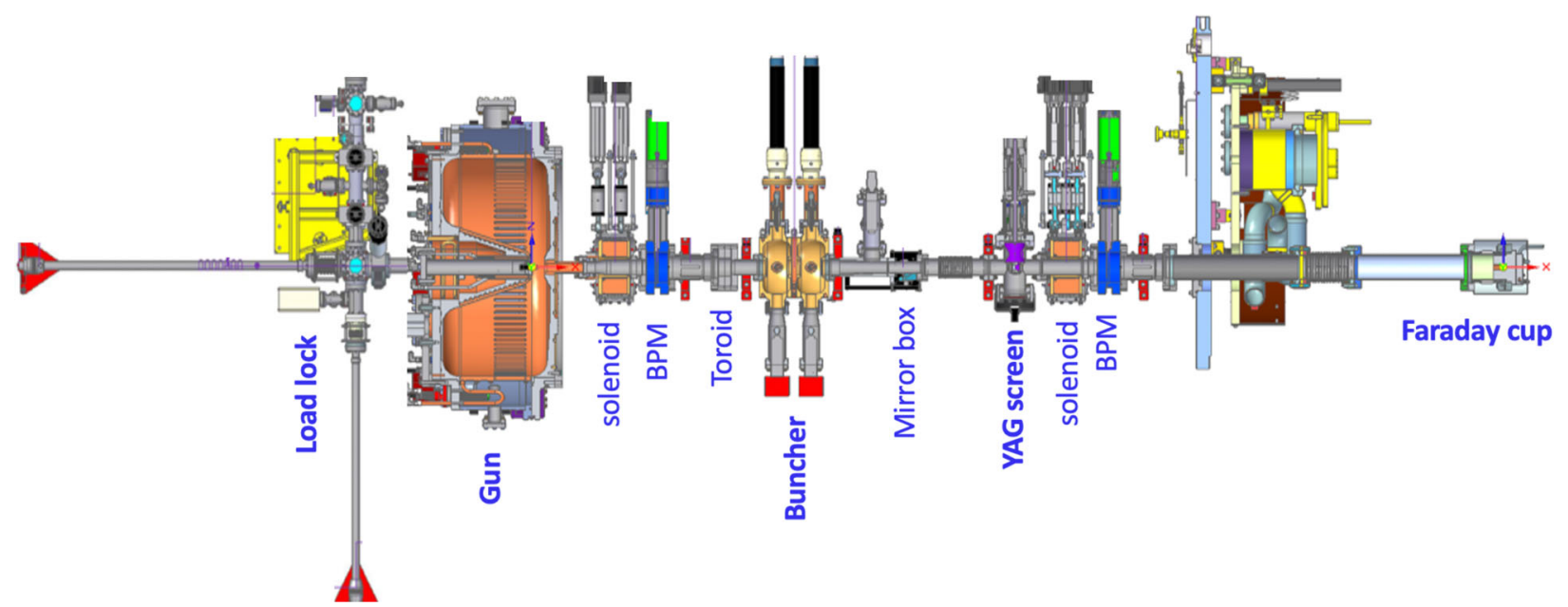

FIG. 1. Schematic of the LCLS-II electron source.

of the gun and allows photocathode plugs to be exchanged. A mirror box is located at $1.1 \mathrm{~m}$ downstream of the cathode for injecting 257.5-nm-wavelength laser pulses.

Commissioning of the electron source began in June 2018 and ended in June 2020 when occupancy of the upstream end of the linac tunnel was required to complete the injector installation. Many problems were encountered and solved during this period to meet the commissioning goals. This paper first discusses the gun, buncher, and laser systems and then presents commissioning results on topics including $\mathrm{cw}$ rf operation, vacuum levels, low-level radio frequency (LLRF) cavity field control, gun and buncher energy gains, megahertz rate bunch generation, intrinsic emittance, and gun dark current characterization. The last section provides a summary of the results.

\section{GUN, BUNCHER, AND LASER SYSTEM}

\section{A. Gun and buncher}

The single-cell gun cavity is designed for cw operation at 185.7 MHz, the seventh subharmonic of the $1.3 \mathrm{GHz}$ superconducting (SC) linac frequency. The cavity has an $\mathrm{R} / \mathrm{Q}=198.2 \Omega, \mathrm{a} \mathrm{Q}_{0}=29923$, and two rf power coupling ports with combined coupling $\beta=1.1$. The design gun voltage gain is $750 \mathrm{kV}$, although it was typically run during commissioning with a voltage gain near $700 \mathrm{kV}$, which nominally requires an input power of $82 \mathrm{~kW}$ and corresponds to a cathode surface gradient of $18.8 \mathrm{MV} / \mathrm{m}$. The rf connection to each of the two-gun ports consists of an airto-vacuum window followed by a $90^{\circ}$ bend that terminates in a loop coupler, all implemented in coaxial waveguide as shown in Fig. 2. The bend keeps dark current from the gun interior from directly hitting the vacuum window. In each feed line, three permanent solenoid magnets (not shown in the plot) are installed in the short section between the rf coupler and the vacuum window to suppress multipacting. Light detectors that view the rf windows and gun body were installed to shut off the rf in the event of an arc, but none has occurred yet.

Each of the gun ports is powered by a $60 \mathrm{~kW}$ solid state amplifier (SSA). In each SSA, power from pairs of laterally diffused metal-oxide semiconductor transistors are first combined locally and then summed in a 30-to-1 coaxial combiner. For reliability, the dc power supplies are distributed within the units: Three $2.5 \mathrm{~kW}, 50 \mathrm{~V}$ supplies are used to power modules containing four transistors. If one module fails, the SSA can still provide $>50 \mathrm{~kW}$ of power.

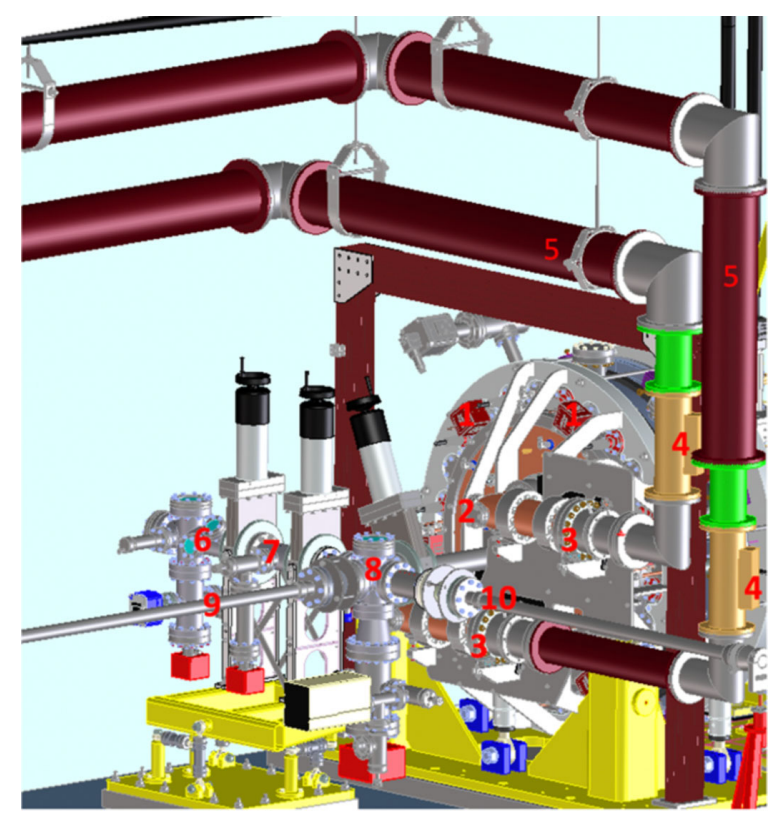

FIG. 2. Layout upstream of the gun showing the waveguide connections and the cathode load-lock system. 1, NEGs; 2, elbow waveguide to rf coupler; 3 , rf window; 4 , rf directional coupler; 5, 6.125-inche waveguide; 6, load-lock suitcase; 7, load-lock airlock; 8, load-lock center chamber; 9 , longitudinal manipulator; 10 , transverse manipulator. 
The SSA output power is very stable in open loop, about $0.01 \% \mathrm{rms}$ in amplitude and $0.1^{\circ} \mathrm{rms}$ in phase on a one second timescale [12]. The SSAs are located in the SLAC linac gallery, and 6.125-inch rigid coaxial waveguide is used to transport the rf power from the SSAs to the gun in the linac tunnel. Although the SSA transistors are back terminated, high-power isolators are included in the transport lines in the gallery. For nonionizing radiation safety, the gallery waveguide is pressurized at a few pounds per square inch, which requires the use of coaxial air barriers. The rf will shut off if there is a drop in pressure inside the waveguide.

With the large size of the gun cavity, there is a substantial frequency change due to $\mathrm{rf}$ heating, about $250 \mathrm{kHz}$ compared to the $6 \mathrm{kHz}$ cavity half-bandwidth. A system of four mechanical tuners is used to push or pull the anode plate to adjust the cavity frequency. For each tuner, a dc motor plus a 30-to-1 gear box rotates a shuttle that screws onto a bolt connected to the anode plate. When the gun warms up, the anode plate bows outward, and the reactive force on the shuttle disk is transferred through a piezo actuator and load cell to a thick plate that is attached to the outer rim of the gun anode. The load cells provide a measure of the applied force, which is adjusted as part of a feedback loop that regulates the cavity frequency. During the warm-up period, the LLRF system employs a selfexcited loop (SEL) controller to track the cavity frequency until it begins to stabilize, and then the tuners are used to set and maintain the cavity frequency near the nominal 185.7 MHz value. The piezo actuators were meant to be used for fine frequency control, but they were not used during commissioning due to reliability issues and their limited range. Upgrades to the tuner system are underway to allow a more linear control and to improve reliability.

The gun vacuum is maintained by six nonevaporable getters (NEGs) and six combined NEGs and ion pumps that are located around the outer cavity radius and connect to a volume that is cut off to the rf (see Fig. 2). A total vacuum pressure of less than $10^{-9}$ Torr, and a partial pressure of less than $10^{-11}$ Torr for specific harmful molecules like oxygen, are required to achieve reasonably long lifetimes of the $\mathrm{Cs}_{2} \mathrm{Te}$ photocathodes used in the gun.

The gun is cooled via five separate water circuits that connect to the SLAC $30^{\circ} \mathrm{C}$ low-conductance water system. The combined flow is about $40 \mathrm{gpm}$, and the flow through the anode plate was adjusted so the steady-state cavity frequency was in a range that did not overstress the tuners.

The rf buncher [13] is a two-cell, $\pi$-mode, $1.3 \mathrm{GHz} \mathrm{NC}$ cavity with $R / Q=340 \Omega$ and $\mathrm{Q}_{0}=25700$. It has four input power couplers with a combined coupling coefficient of unity. The cavity adds an energy chirp to the bunches, and the resulting velocity variation compresses the bunch length by a factor of 3-5. Each input coupler is powered by a $3.8 \mathrm{~kW}$ SSA. For the nominal combined input of power of $7.8 \mathrm{~kW}$, the integrated buncher field is expected to be
$260 \mathrm{kV}$. The buncher LLRF system capabilities are similar to that for the gun. A SEL controller is used to track the cavity frequency during warm-up, and a dedicated chiller is used to regulate cavity frequency once it is close to the nominal frequency. The rf heating detunes the cavity by about $600 \mathrm{kHz}$, much larger than its $50 \mathrm{kHz}$ halfbandwidth.

\section{B. Laser system}

The laser system uses an infrared (IR) front end that was manufactured by Amplitude Systems Inc. Main components include an ytterbium-based fiber oscillator operating at $46.43 \mathrm{MHz}$, a pulse picker to reduce the pulse frequency from 46.43 to $1 \mathrm{MHz}$, a diode-pumped fiber amplifier (Tangerine), an acousto-optics modulator to select the laser rate, and a compressor. The oscillator is phase locked to the accelerator phase reference frequency. The IR front end produces $50 \mu \mathrm{J}, 1030 \mathrm{~nm}$ pulses with an adjustable repetition rate up to $1 \mathrm{MHz}$ and an adjustable pulse duration from $\sim 330$ fs to $30 \mathrm{ps}$.

For the IR to ultraviolet (UV) conversion, a combination of two critically phase-matched barium borate (BBO) crystals are employed. The first frequency doubling stage is a 3-mm-thick second harmonic generation (SHG) BBO crystal that generates $515 \mathrm{~nm}$ pulses, and the second stage is a 1-mm-thick fourth harmonic generation (FHG) $\mathrm{BBO}$ crystal that produces the $257.5 \mathrm{~nm}$ pulses required for cathode photoemission. The FHG crystal is located in a temperature-regulated enclosure. For the temporally compressed (near transform limit) IR pulse SHG, the conversion efficiency is $50 \%-65 \%$, and the FHG conversion efficiency from IR to UV is $8 \%-20 \%$ [14]. The efficiencies depend on the IR repetition rate and, thus, the thermal loads in the SHG and FHG crystals.

While compressed IR pulses yield the highest conversion efficiency and the shortest pulse duration in the UV, the photoinjector beam parameters require UV pulses with longer temporal durations. Introducing a chirp into the IR beam in a traditional collinear SHG and FHG stage can produce temporal intensity modulations in the UV beam and yield insufficient conversion efficiency. Instead, our approach involves FHG using the fully compressed IR beam and utilizing the maximum available UV bandwidth with a two-pass reflective grating stretcher. The stretcher path length is adjustable to vary the pulse duration in the present configuration from 10 to $30 \mathrm{ps}$ FWHM. The firstorder efficiency of the grating is about $80 \%$, yielding about $40 \%$ transmission through the two-pass stretcher [14].

The high power of the laser, the substantial UV absorption, and the low thermal conductivity of BBO cause severe thermal management challenges in the FHG crystal. At repetition rates above $100 \mathrm{kHz}$, we initially observed very unstable UV power, poor beam quality, and strong thermal lensing. We resolved this problem by enlarging the beam size in the FHG crystal at each power level using a zoom 
telescope. By increasing the beam diameter from 1.5 (for a repetition rate $<10 \mathrm{kHz}$ ) to $2.8 \mathrm{~mm}$ (for $1 \mathrm{MHz}$ ), we achieved stable power levels up to $1 \mathrm{MHz}$ with the sacrifice of conversion efficiency and a smooth Gaussian pulse spatial shape at all repetition rates. However, even with the reduced $1 \mathrm{MHz}$ rate conversion efficiency, we were able to meet the final pulse energy specification of $\sim 0.3 \mu \mathrm{J}$ on the photocathode, which is required if the quantum efficiency (QE) is as low as $0.5 \%$.

The laser beam is transported to the accelerator tunnel through about $20 \mathrm{~m}$ of evacuated $\left(10^{-7}\right.$ Torr $)$ pipe. The laser output plane is imaged onto the iris, which is located on the optical table next to the gun. This iris is overfilled by the laser beam, which creates the required hard-edge spatial shape when imaged at the cathode.

\section{COMMISSIONING RESULTS}

\section{A. Gun vacuum processing}

The original plan was to bake the large volume gun by applying rf power with no gun water cooling. However, severe multipacting due to significant amounts of hydrocarbons in the gun prevented increasing the rf power above about $600 \mathrm{~W}$. Figure 3 (left) shows a residual gas analyzer (RGA) display at the time. The 12 NEGs were eventually identified as the source of the contamination, as it was later found they had been processed in an oil-contaminated chamber during assembly. We instead thermal baked the gun at up to $190^{\circ} \mathrm{C}$ for more than 10 days, but no notable reduction in the hydrocarbons was observed, and severe multipacting still occurred when rf was applied. This was followed by a long period of thermal conditioning that included a slow activation of the 12 NEGs. This process significantly reduced the hydrocarbon partial pressures, and we were able to power the gun to $80 \mathrm{~kW}$ without significant multipacting [15]. Multipacting at lower energy [16] was observed, but it was easy to be processed. The gun was operated $\mathrm{cw}$ for about $700 \mathrm{~h}$ for the commissioning program, during which the vacuum pressure continually

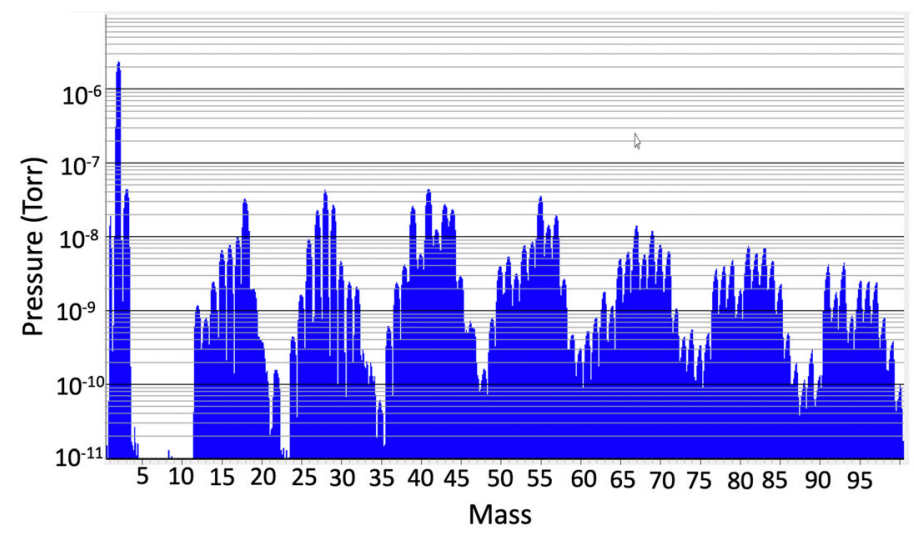

decreased, reaching $8 \times 10^{-10}$ Torr at nominal rf power. Figure 3 (right) shows the corresponding RGA scan where the partial pressures of the major mass components are in the $10^{-11}$ Torr scale at nominal rf power. With the rf off, the gun vacuum pressure level has been as low as $3 \times 10^{-11}$ Torr.

\section{B. Gun and buncher cw operation}

Gun operation at nominal power and frequency was routinely achieved after issues with the gun tuners were solved [15]. The turn-on process involved several steps. When rf power was first applied, the LLRF system was operated in SEL mode in which the drive frequency tracks the cavity frequency. The rf duty cycle was increased in $10 \%$ increments over several minutes until it was above 99\%. In pulse mode, the cavity frequency is inferred from the measured frequency of the rf discharged from the cavity during the period when the drive rf is off. The duty cycle had to be increased gradually, as the frequency-tracking algorithm was limited to rate changes of about $1 \mathrm{kHz} / \mathrm{s}$; otherwise, the increased detuning would cause significant rf reflections that would trip off the rf due to multipactorinduced vacuum activity in the couplers [12]. This rate limitation is being remedied by an upgrade to LLRF system firmware.

During the power ramp-up period, there was an $\sim 350 \mathrm{kHz}$ cavity frequency increase due to the rf heating of the anode plate, which bowed it outward. Once this change began to level off, the mechanical tuners were manually adjusted to bring the cavity within a few hundred hertz of the nominal frequency. SEL mode was then turned off, and a two-tiered control loop was turned on in which a high-level feedback loop adjusted the tuner load cell set points based on the detuning relative to the nominal frequency, and four low-level feedback loops regulated the four tuner loads to their set point values. This feedback was critical, as there was a subsequent $-100 \mathrm{kHz}$ frequency drift with a several-hour time constant due to the slow rf heating of the outer cavity wall. During this period,

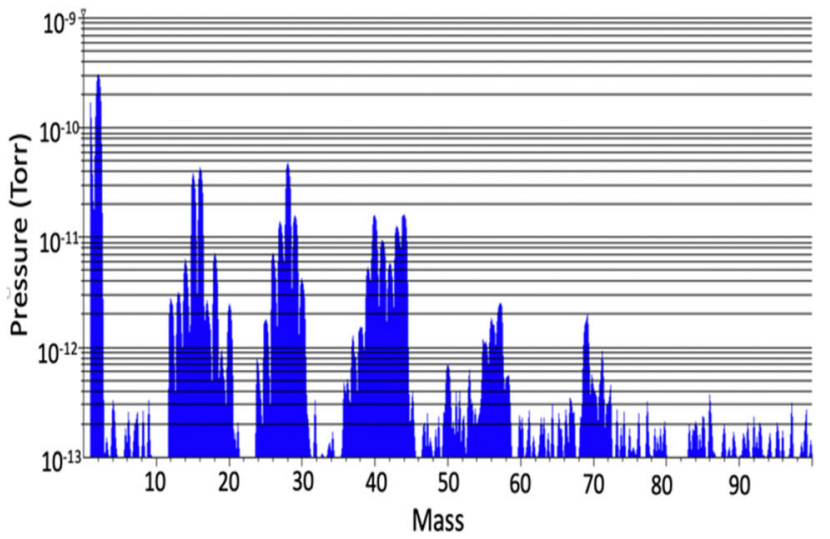

FIG. 3. Left: early gun RGA spectrum showing significant hydrocarbon contamination. Right: improved gun vacuum at nominal cw rf power after NEG activation. 

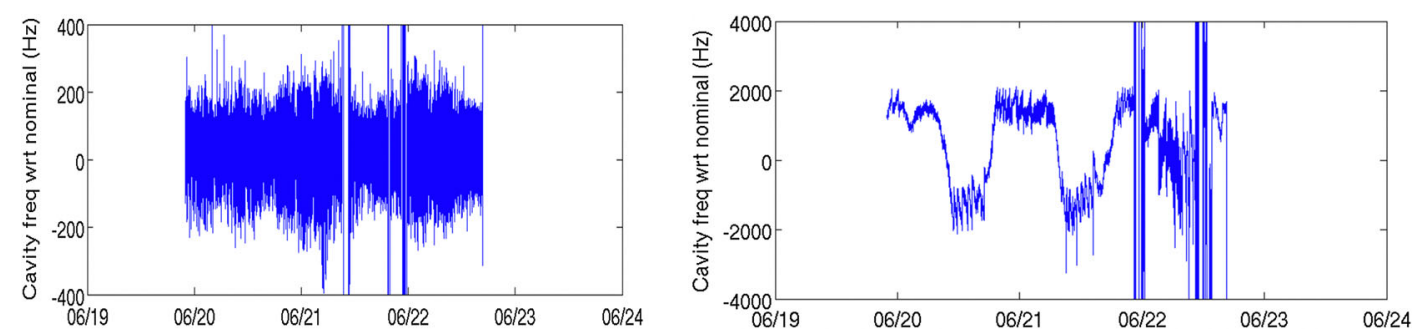

FIG. 4. Gun (left) and buncher (right) cavity detuning versus time (in days) when operating at nominal power and frequency with the frequency feedback on.

the duty cycle was increased to $100 \%$, and cavity frequency was then computed based on the phase difference between the forward and probe signal rf. This control procedure regulated the frequency to within about $200 \mathrm{~Hz}$ of the nominal value, which is $\sim 3 \%$ of the cavity half-bandwidth. Figure 4 (left) shows a few days history of the frequency regulation. The gun vacuum improved by about a factor of 3 when switching from 99\% duty to $\mathrm{cw}$. The higher pressure in pulse mode was likely the result of multipacting-induced outgassing, which occurs at the lower power levels witnessed during the pulse rise and fall times.

We also established cw operation for the buncher cavity at nominal power and frequency. The buncher turn-on procedure was similar to that for the gun except that a different method was used for adjusting the cavity frequency, as discussed below. As with the gun, the buncher LLRF system was run in frequency-tracking mode (SEL), while the buncher input power was ramped up and the duty factor was increased to above $99 \%$. Near steady state, when the frequency was close to $1.3 \mathrm{GHz}$, the detuning calculation method was switched from decay-waveform-based, which can be done only in pulse mode, to forward-toprobe-phase based, and then the duty factor was increased to $100 \%$. The frequency was then locked to $1.3 \mathrm{GHz}$, and the buncher water temperature was varied as part of a feedback loop to regulate the cavity frequency. Figure 4 (right) shows an example of the detuning variation during $\mathrm{cw}$ operation at nominal power (for reference, $2 \mathrm{kHz}$ is $4 \%$ of the buncher half-bandwidth). Getting this regulation to work proved difficult initially, as the water chiller, which had its own feedback control, caused the water temperature to oscillate about its set point. Using a higher-capacity chiller solved this issue.

\section{Gun and buncher field regulation}

Regulation of the gun and buncher field amplitude and phase is critical to limit beam arrival time jitter at the end of the linac. Table II lists the regulation requirements for attaining low (femtosecond-level) timing jitter after two stages of bunch compression.

The LLRF systems that were used to drive the gun and buncher SSAs are based on the design used at LBNL [17], which does not have all of the features that have been incorporated in the version that will regulate the SC linac cavity fields. These features will be implemented before electron source operation resumes in late 2021. For the source commissioning studies, however, regulation of the gun and buncher amplitude and phase was not necessary, as the open loop SSA rf output power was sufficiently stable. Nonetheless, tests were done to demonstrate that the LLRF PI-based feedback control as implemented could achieve the required stability specs over a period of hours. Figure 5 shows rms amplitude and phase jitter measurements from such a regulation test for the gun (left) and buncher (right), respectively. For the gun, the rms jitter shown is that of the cavity probe signal being regulated (i.e., the LLRF feedback system varies the gun input rf amplitude and phase to stabilize this signal: An independent measure of the cavity field stability was not available at the time), while for the buncher, it is the jitter from an independent cavity probe signal, that is, one that is not being directly regulated. In all cases, the average rms jitter meets the specs in Table II.

\section{Gun and buncher energy and phase measurements}

We used five methods to measure the beam energy from the gun. The first method was based on the signals from the two rf probes that are installed on the gun body. The second method was based on the measured input power to the gun, which relies on factory-calibrated directional couplers. Both of these methods require knowledge of the cavity rf parameters: R/Q was computed from the gun geometry, and $Q_{L}$ and the probe and power coupler betas were measured in situ with a network analyzer. The third method was to measure the electron momentum by its deflection on the YAG screen caused by a change in a corrector dipole magnet setting (i.e., from the slope of the position versus corrector strength). The fourth method was based on the upstream solenoid setting that produced the minimum

TABLE II. Gun and buncher field regulation specifications.

\begin{tabular}{lc}
\hline \hline & Specification \\
\hline Gun rms amplitude & $<0.01 \%$ \\
Gun rms phase & $<0.04^{\circ}$ \\
Buncher rms amplitude & $<0.03 \%$ \\
Buncher rms phase & $<0.015^{\circ}$ \\
\hline \hline
\end{tabular}



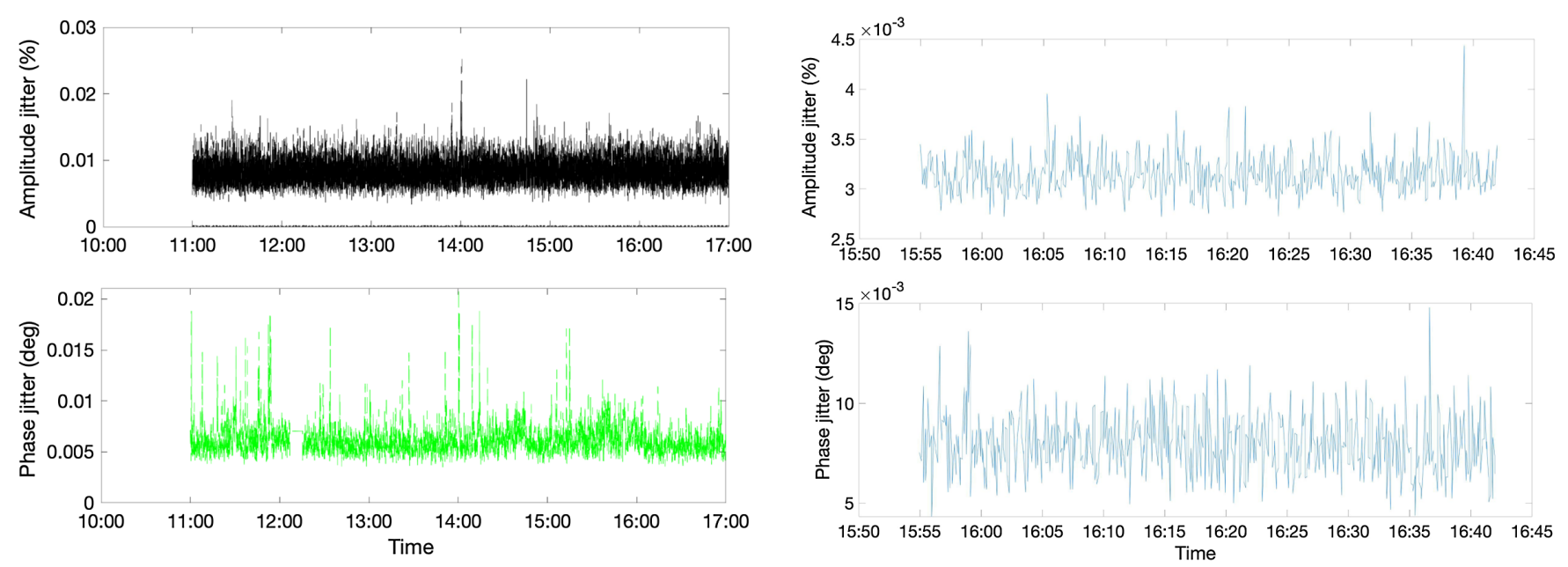

FIG. 5. Gun (left) and buncher (right) probe signal amplitude and phase jitter versus time with the feedback on.

beam size on the YAG screen for low-charge $(\sim 1 \mathrm{pC})$ bunches. The final method was based on measuring the beam time of flight using the signals from the two BPMs. These five methods yielded beam energies that agree within about $10 \%$ for the same cavity input power. As a measurement example, Fig. 6 (left) shows the inferred gun energy gain versus gun phase based on the dipole corrector method. The zero phase in this plot was determined by observing the phase at which the charge of the bunch goes to zero. During LCLS-II operation, the beam will be nominally generated close to the rf crest, which yields the lowest emittance.

The buncher if amplitude and phase were determined by measuring the beam energy versus buncher if phase as shown in Fig. 6 (right) (the gun input power and phase were held constant during the scan). The inferred gun and buncher energy gains from the sine-wave fit to the data are 744 and $237 \mathrm{keV}$, respectively, which agree reasonably well with expectations based on the rf input power at the time. The buncher phase will nominally be set to a zero crossing to bunch the beam, which, without knowing the absolute sign of the phase, could be either point A or B in Fig. 6 (right). ASTRA [18] simulations show that the beam size at the zero-crossing phase for bunching is much larger than that for debunching due to space charge effects [7]. Figure 7 shows electron beam images on the YAG screen at the two zero-crossing phases for an $\sim 40 \mathrm{pC}$ bunch charge. The larger size at zero crossing $\mathrm{B}$ indicates that it was bunching the beam.

To verify our understanding of the beam energy and optics, we compared beam size measurements with predictions. First, we turned off the buncher and measured the rms beam size at the YAG screen. Given the solenoid strength, bunch charge, laser pulse length, and laser size on the cathode, the measured beam size was reproduced by an ASTRA simulation that assumed a gun energy gain of $710 \mathrm{keV}$, close to that measured with the dipole method. We then turned on the buncher and measured the rms beam size at the YAG screen versus the buncher phase as shown in Fig. 8. These measured sizes match the ASTRA
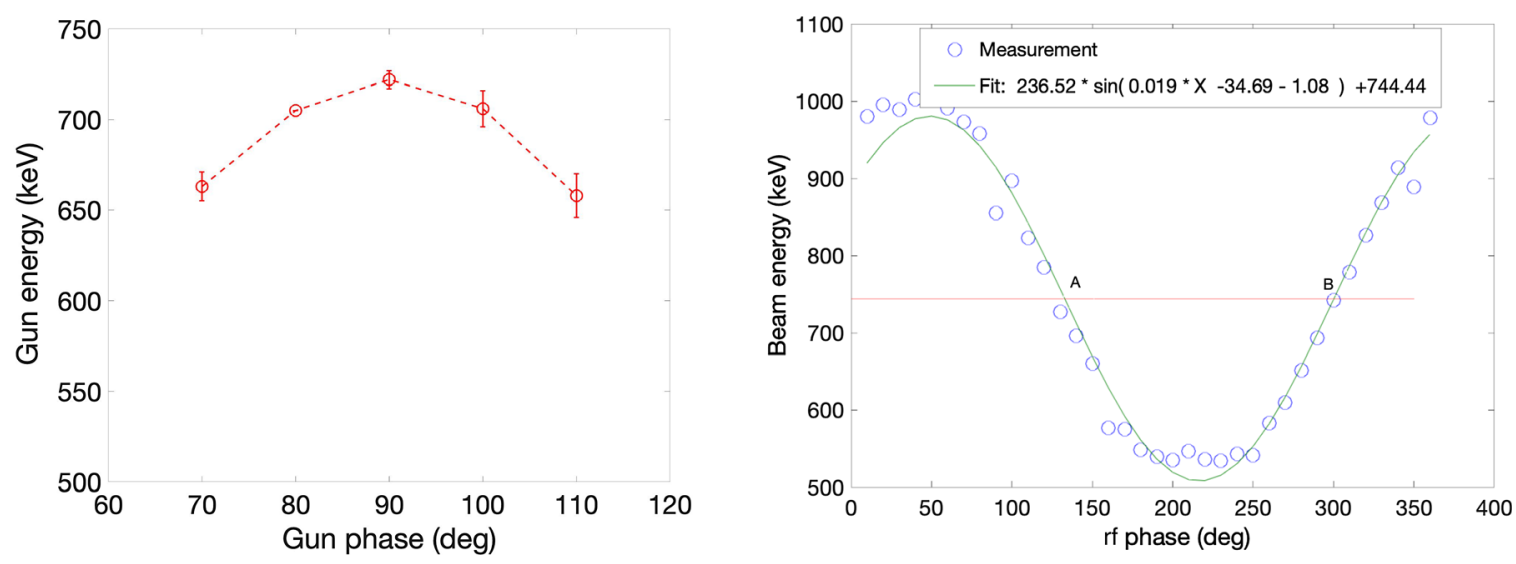

FIG. 6. Left: measured gun energy versus gun rf phase. Right: energy scan versus buncher rf phase. Points A and B are the buncher zero-crossing phases. 

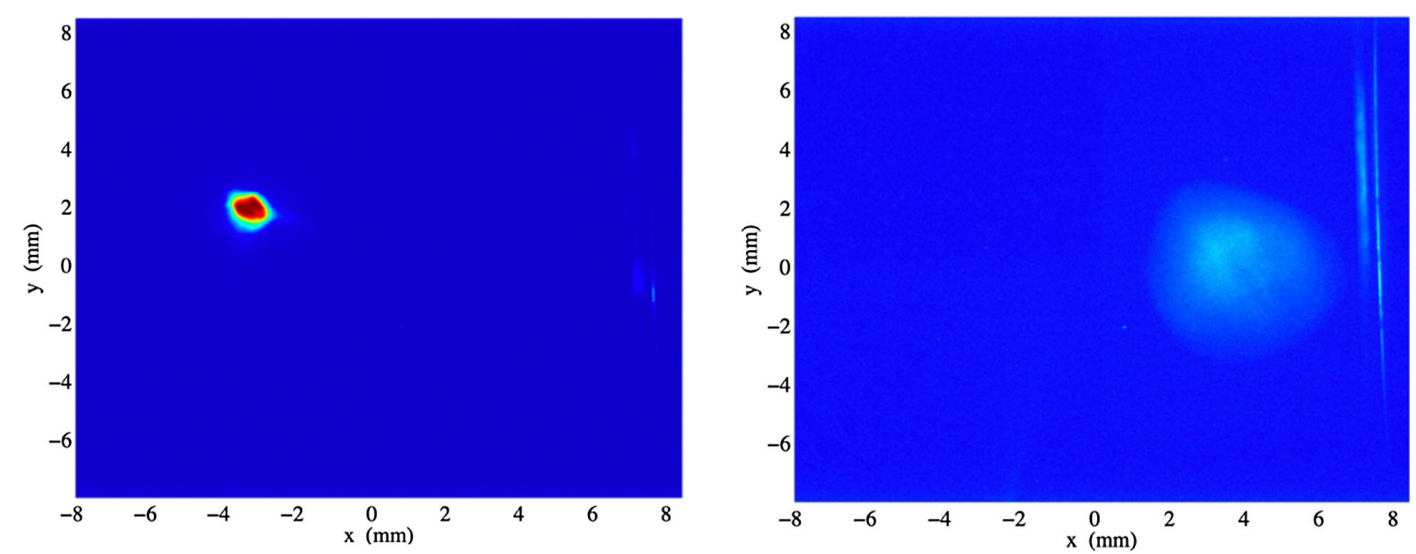

FIG. 7. Measured beam sizes for $40 \mathrm{pC}$ bunches at zero crossing A (left) and B (right). The larger size at zero crossing B indicates that the beam was being bunched.

simulations fairly well assuming a buncher energy gain of $225 \mathrm{keV}$, which is close to that expected.

\section{E. Demonstration of $30 \mu \mathrm{A}, 1 \mathrm{MHz}$ bunch operation}

LCLS-II requires a bunch repetition rate up to about $1 \mathrm{MHz}$. At this rate, the bunch current will initially be kept below $30 \mu \mathrm{A}$ due to the heat load limitation in the beam dump at the end of the LCLS-II linac. Figure 9 shows that the gun can deliver this current and bunch rate. The beam current was measured using the Faraday cup at the end of the electron source beam line (see Fig. 1). A picoamp meter measured the current, and the dark current contribution was subtracted to obtain the data shown in Fig. 9. Note that, at a laser rate of $100 \mathrm{~Hz}$ and lower, the dark current was higher than photocurrent and varied over time. Thus, the inferred photocurrent has a larger error at low rates. The roll-off of the current above $100 \mathrm{kHz}$ was due to a drop in the laser pulse energy as a result of component heating. The photocathode QE for these measurements was 10 times

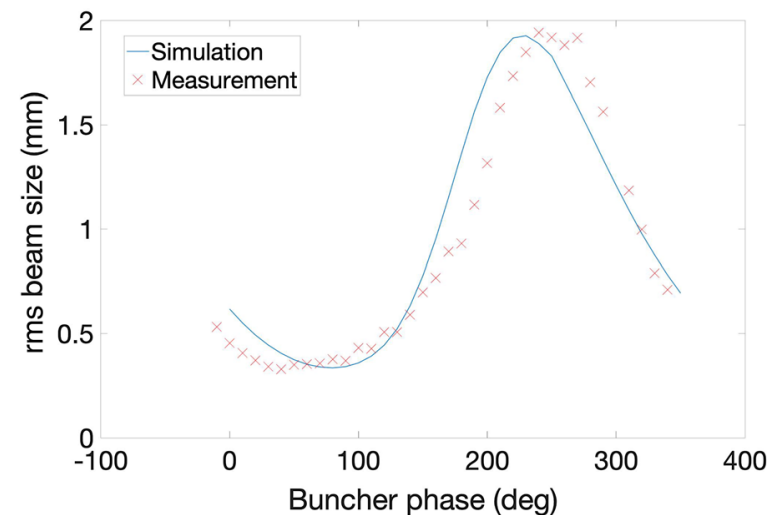

FIG. 8. Measured beam size versus buncher phase. For a buncher energy gain of $225 \mathrm{keV}$, the ASTRA simulations (blue curve) are a fair match to the data, which were taken with $40 \mathrm{pC}$ bunches and 1-mm-diameter laser pulses with a 20 ps FWHM Gaussian temporal profile. lower than nominal, so heating will be less of an issue with our newer, higher-QE cathodes.

\section{F. $\mathrm{Cs}_{2} \mathrm{Te}$ cathode intrinsic emittance}

The intrinsic emittance of the photocathode is one of the key parameters of a high-brightness electron source. $\mathrm{Cs}_{2} \mathrm{Te}$ cathodes produced by INFN and LASA were used for the electron source commissioning. The emittance measurements were made by varying the upstream solenoid strength and recording the beam size on the YAG screen for a beam energy of about $700 \mathrm{keV}$ with the buncher off. A fit to these data yields the beam emittance and Twiss parameters just upstream of the solenoid [19]. The space charge contribution was minimized by using a low bunch charge $(<1 \mathrm{pC})$ with $\sim 20$ ps FWHM Gaussian laser pulses. Figure 10 shows the resulting normalized horizontal emittances versus the rms laser spot size as measured on the virtual cathode screen. A linear fit to the data yields a $1.0 \pm$ $0.1 \mu \mathrm{m} / \mathrm{mm}$ slope, close to the theoretical expectation using the formula in Ref. [20] for the $4.84 \mathrm{eV}$ UV laser photon energy and assuming a 3.2-3.5 eV work function for $\mathrm{Cs}_{2} \mathrm{Te}$ [21].

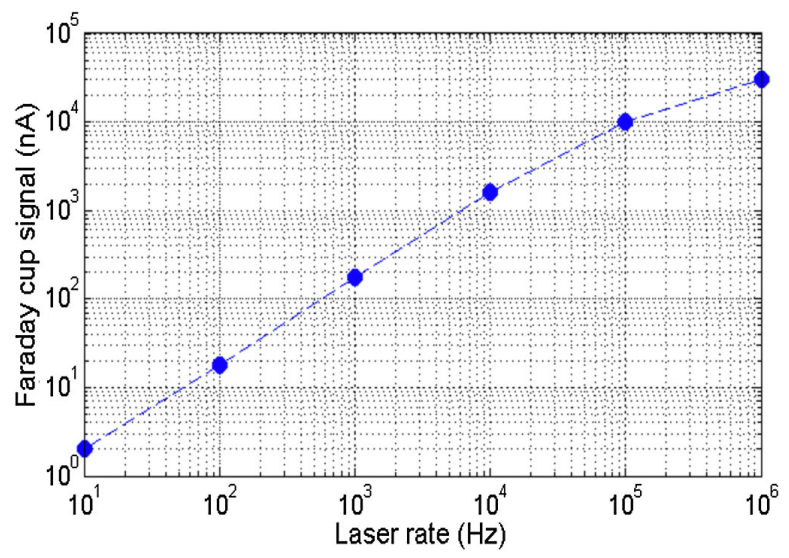

FIG. 9. Electron beam current versus laser pulse rate. 


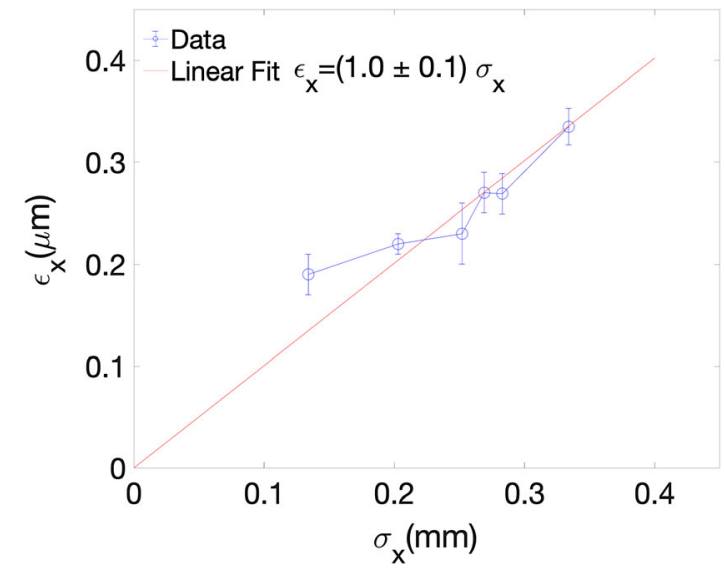

FIG. 10. Measured horizontal normalized emittance versus the rms laser spot size for low-charge bunches.

\section{G. $\mathrm{Cs}_{2}$ Te photocathode QE}

The quantum efficiencies of the two $\mathrm{Cs}_{2} \mathrm{Te}$ photocathodes that were used during commissioning were less than $0.5 \%$, whereas around $5 \%$ was expected. This degradation likely occurred as result of a small vacuum leak in the transport container during their handling at SLAC, which has since been fixed. In addition, the QE near the center of the first installed photocathode decreased significantly during the course of about $500 \mathrm{~h}$ of gun operation when the beam was typically operated at $10 \mathrm{~Hz}$. Operation at high rate was less than $30 \mathrm{~min}$ in total, as radiation-induced charging of the uncoated Faraday cup ceramic led to arcing. Figure 11 (left) shows the QE map of the photocathode measured just before it was removed-a craterlike QE degradation is readily discernible. The figure also includes a photograph of the cathode after it was removed, which shows a discolored region corresponding to the QE crater (the surface appearance was also affected by its exposure to air after removal).
The second photocathode witnessed about $200 \mathrm{~h}$ of gun operation where again the beam rate was mostly $10 \mathrm{~Hz}$. Several QE maps were made during this period and showed that the QE decreased by about a factor of 2 near the center of the photocathode relative to that near the perimeter. When the photocathode was removed, a discolored region similar to that in the first photocathode was observed, although it was less pronounced. For both cathodes, XRF and XPS analyses of the $\sim 80$-nm-thick $\mathrm{Cs}_{2}$ Te layer are being performed to understand the changes that occurred in the discolored regions.

Given the low beam rate operation, ion back bombardment is unlikely to be the cause of the QE degradation. Also, transport simulations of beam-generated ions show that only those produced within a few millimeters of the cathode would impinge on it - the rest migrate downstream. Dark current may be a factor and is discussed in the next section. Although the photocathode QE may degrade over time, the baseline plan allows for the cathode plug to be exchanged every few weeks, so operational availability should not be impacted.

\section{H. Dark current measurements, analysis, and mitigations}

Dark current emitted from the gun is a concern if it is accelerated in the linac and lost near the undulators, which are very sensitive to radiation damage. We measured the dark current level with the Faraday cup, and, for monitoring purposes, we used the corrector magnets to maximize the value-the upstream solenoid focusing was weaker than nominal during these measurements. Initially, the dark current was about $10 \mathrm{nA}$ but gradually grew to the $\mu \mathrm{A}$ level during the $\sim 700 \mathrm{~h}$ of $\mathrm{cw}$ gun operation. Figure 12 (left) shows a YAG screen image of both a laser-generated beam and the dark current, which is dominated by emission from two sites. Based on the cathode plug geometry and the beam to dark current separation on the YAG screen, the
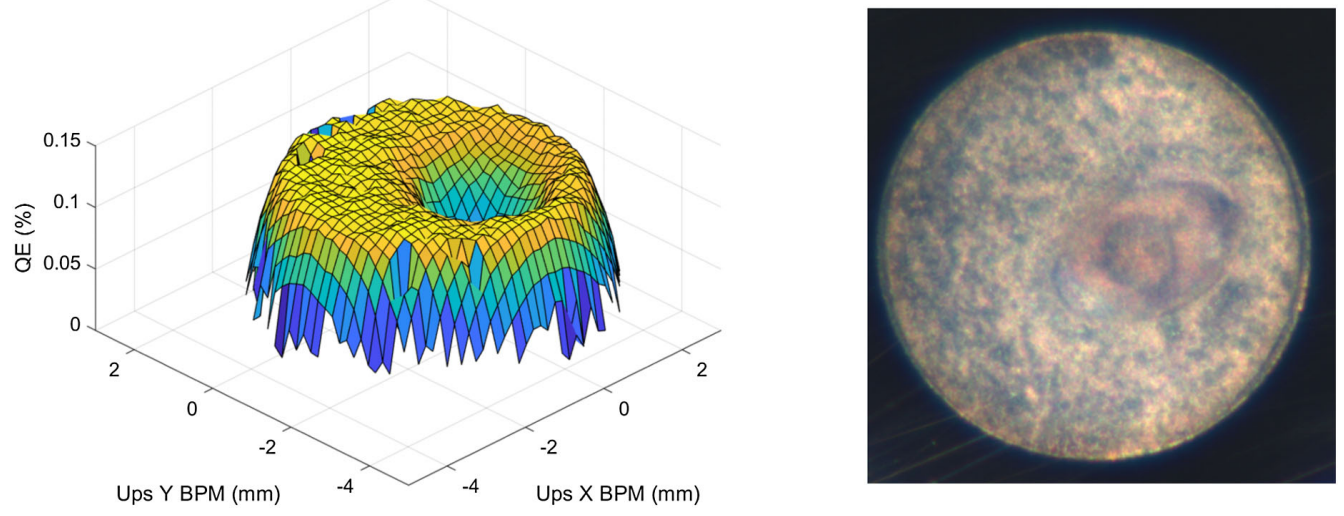

FIG. 11. Left: QE map of the first $\mathrm{Cs}_{2}$ Te photocathode after $500 \mathrm{~h}$ of operation as measured by recording the bunch charge versus downstream beam position as the UV laser was scanned over the cathode surface. Right: photo of the 5-mm-diameter photocathode after it was removed and exposed to air. 

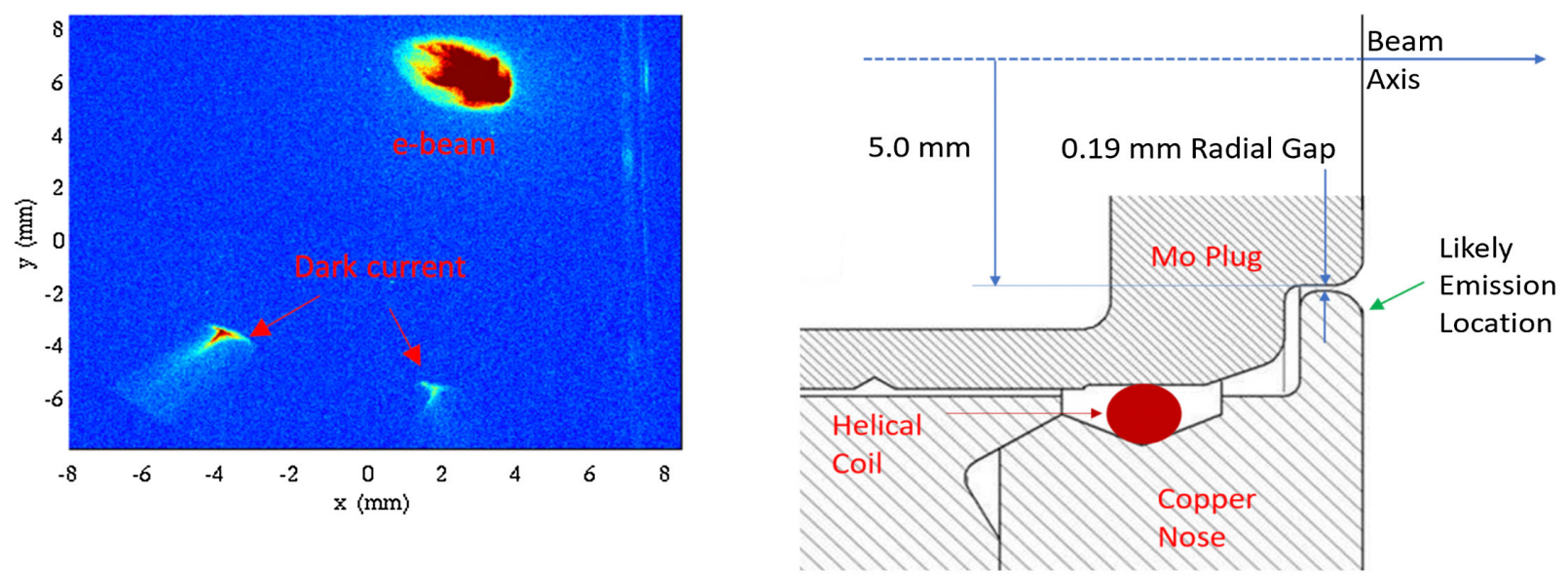

FIG. 12. Left: YAG screen image of a laser-generated electron beam and dark current. Right: geometry of the molybdenum cathode plug when inserted into the copper cathode nose.

field emission sites are likely near the rounded opening on the copper cathode nose, where the electric field is enhanced by $24 \%$ relative to that at the center of the plug [22] [see the diagram in Fig. 12 (right)]. This is consistent with the fact that the dark current level did not change when the cathode plugs were swapped. Photos of the cathode nose taken through a port in the mirror box show no obvious features, such as scratches, corresponding to these two emission spots. Similar emission sites were observed in the APEX gun at LBNL [23]. No discernible dark current was measured from the buncher cavity, which has much lower surface fields.

Although the maximum dark current at the Faraday cup grew to about $2 \mu \mathrm{A}$, only $\sim 0.5 \mu \mathrm{A}$ is expected to enter the first cryomodule beam aperture based on our measurements with nominal beam optics and steering. This level essentially meets our spec of $<0.4 \mu \mathrm{A}$ to limit downstream radiation. Still, we are looking at mitigations to reduce the transmitted current, as it is uncertain whether it will continue to increase. One approach is to dry-ice clean

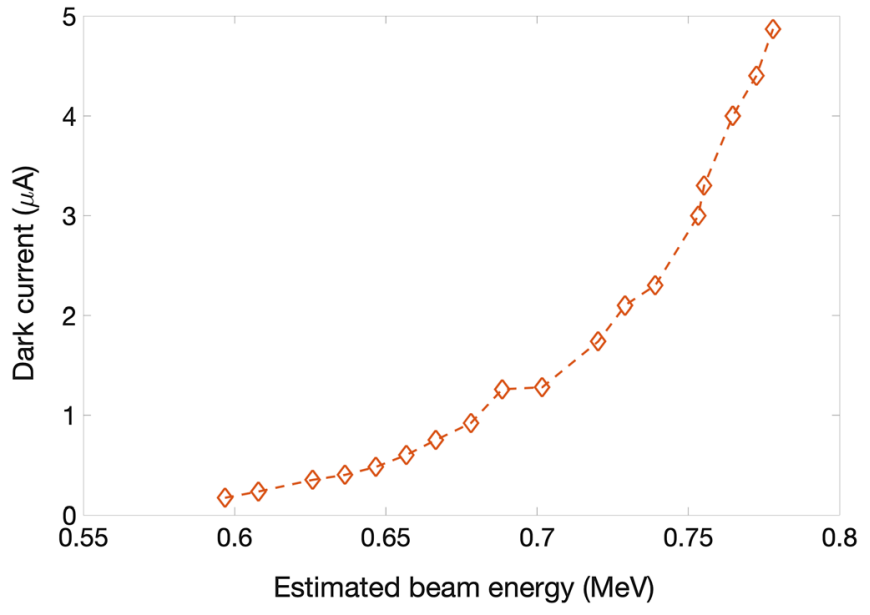

FIG. 13. Measured dark current versus beam energy. the gun cavity in situ, but this would be fairly invasive at this stage, as would be plasma processing. Another method is to use collimation, which will be implemented before operation resumes by including an array of apertures in the YAG screen insertion mechanism. FLUKA [24] simulations show that the radiation from the collimator at the downstream cryomodule and the upstream gun should be minor [25]. Finally, we could operate with a lower gun energy, which lowers the dark current as shown in Fig. 13. Operating at $650 \mathrm{keV}$ instead of $750 \mathrm{keV}$, for example, would reduce the dark current by a factor of about 3 . Simulations show that the lower gun energy in this case would produce a negligible change in the optimized beam emittance at the end of the injector for the nominal $100 \mathrm{pC}$ bunches.

In the long term, a spare gun is being built for LCLS-II that will have an elliptical-shaped plug gap opening to reduce the field enhancement and a stainless-steel insert around the nose opening to reduce field emission [22]. No emission sites on the molybdenum plug surfaces have been observed, and stainless steel, which is easier to braze, may prove as robust.

We have also begun studies to identify where dark current electrons impact the cathode and beam apertures and where secondary electrons produced from such interactions end up. Figure 14 shows a GPT [26] transport simulation using the standard scattering option for copper, which is a simple reflection model with random energy loss [26]. Therefore, the relative number of secondaries shown in the figure should be viewed qualitatively (a better secondary yield model is being developed). In this simulation, the primary electrons were emitted from a onemicron radius point source at the location indicated by the green arrow in Fig. 12 (right), which is on the cathode edge, $5 \mathrm{~mm}$ away from the cathode center. As can be seen, the primary dark current (in blue) is largely lost between the buncher and the laser mirror box, although the secondaries 


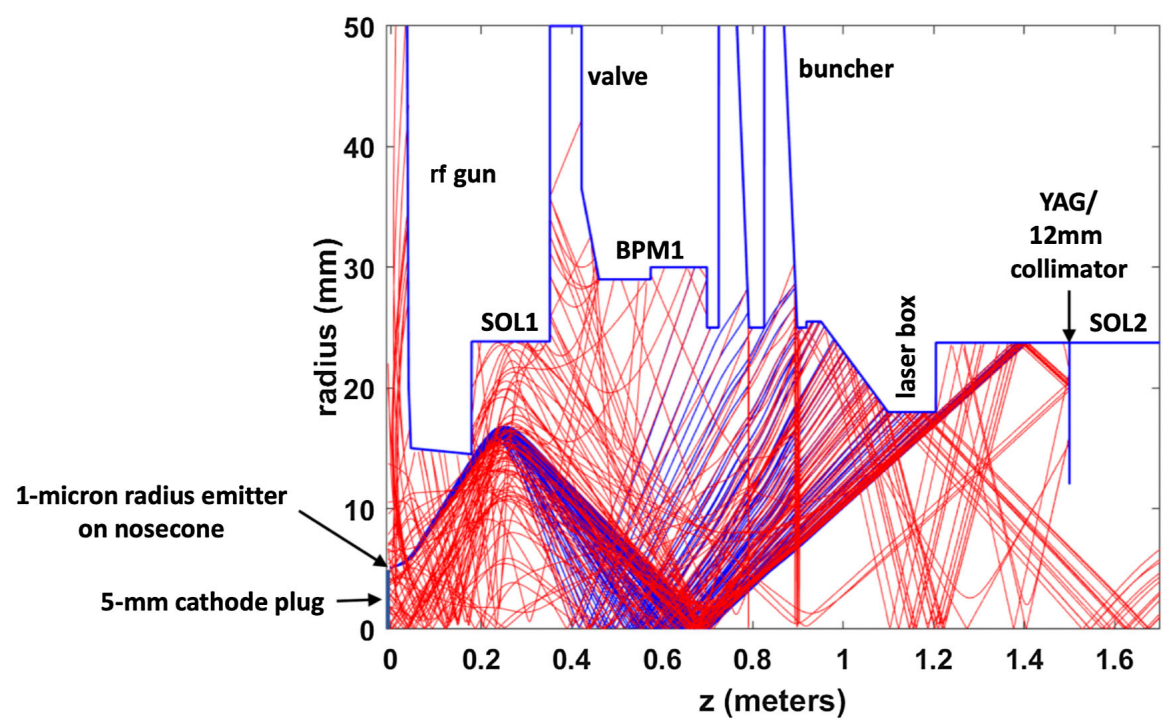

FIG. 14. GPT simulation showing the trajectories of field-emitted primary electrons (blue) and the resulting secondary electrons (red), which mostly propagate toward the gun. The collimator that is shown will be installed before operation resumes.

(in red) propagate farther downstream, and upstream to the cathode. Transport studies have also been done for dark current emitted from the gun anode nose, which naturally propagates toward the cathode. The electric field on the anode nose is similar to that on the cathode. A large emitter on the anode nose could perhaps produce enough current to degrade the QE in the pattern we observe. We have no better explanations at this time.

\section{SUMMARY}

The LCLS-II electron source was successfully commissioned over a two-year period during which the gun was operated $\mathrm{cw}$ for about $700 \mathrm{~h}$. The gun vacuum pressure improved significantly after a slow activation of the NEG pumps to remove hydrocarbon contamination. Operation of gun and buncher at nominal power and frequency became routine after procedures were developed to deal with the large change in the cavity frequencies during warm-up. The energy gain provided by the gun and buncher was in fair agreement with expectations, and the cavity fields could be regulated with the LLRF systems to achieve the desired stability. Although the $\mathrm{Cs}_{2} \mathrm{Te}$ photocathode QEs were lower than expected, the baseline $1 \mathrm{MHz}, 30 \mu \mathrm{A}$ beam was generated, and the photocathode intrinsic emittance met expectation. During operation, QE degradation near the cathode center was observed, which may be dark current related. Mitigations to reduce the dark current are being implemented.

\section{ACKNOWLEDGMENTS}

We thank A. Brachmann, W. Colocho, Y. Ding, B. Dunham, D. Filippetto (LBNL), M. Johnson (LBNL), D. Li (LBNL), T. Luo (LBNL), T. Maxwell, N. Neveu,
S. Virostek (LBNL), M. Woodley, C. Zimmer, and many technical colleagues for fruitful discussions and strong support of the work. Also, we thank INFN and Laboratory for Accelerators and Applied Superconductivity for providing photocathodes for the electron source commissioning. The work is supported by U.S. Department of Energy under Grant No. DE-AC02-76SF00515.

[1] P. Emma et al., Nat. Photonics 4, 641 (2010).

[2] W. Ackermann et al., Nat. Photonics 1, 336 (2007).

[3] H. Tanaka, in Proceedings of the 2nd International Particle Accelerator Conference, San Sebastián, Spain (EPS-AG, Spain, 2011), p. 21.

[4] H. Braun, in Proceedings of the 8th International Particle Accelerator Conference (IPAC 2017), Copenhagen (JACoW, Geneva, 2017).

[5] J. Galayda, in Proceedings of the 9th International Particle Accelerator Conference (IPAC 2018), Vancouver (JACoW, Geneva, 2018), p. 18.

[6] J. Schmerge et al., in Proceedings of the 36th International Free Electron Laser Conference (FEL 2014), Basel, Switzerland (JACoW, Geneva, 2014), p. 815.

[7] F. Zhou et al., in Proceedings of the 37th International Free Electron Laser Conference (FEL 2015), Daejeon, Korea (JACoW, Geneva, 2015), p. 77.

[8] F. Sannibale et al., Phys. Rev. Accel. Beams 15, 103501 (2012).

[9] F. Sannibale et al., in Proceedings of the 7th International Particle Accelerator Conference (IPAC2016), Busan, Korea (JACoW, Geneva, 2016), p. 1041.

[10] R. P. Wells, W. Ghiorso, J. Staples, T. M. Huang, F. Sannibale, and T. D. Kramasz, Rev. Sci. Instrum. 87, 023302 (2016). 
[11] D. Sertore, S. Schreiber, K. Floettmann, F. Stephan, K. Zapfe, and P. Michelato, Nucl. Instrum. Methods Phys. Res., Sect. A 445, 422 (2000).

[12] C. Adolphsen et al., in Proceedings of the 19th International Conference on RF Superconductivity (SRF 2019), Dresden, Germany (JACoW, Geneva, 2019), p. 891.

[13] S. Virostek et al., in Proceedings of the 8th International Particle Accelerator Conference (IPAC2017), Copenhagen, Denmark (JACoW, Geneva, 2017), p. 4380.

[14] S. Gilevich et al., Report No. SLAC-TN-20-003, 2020.

[15] F. Zhou et al., in Proceedings of the 10th International Particle Accelerator Conference (IPAC2019), Melbourne, Australia (JACoW, Geneva, 2019), p. 2171.

[16] T. Huang, CBP Note No. 393, LBNL, 2008.

[17] G. Huang et al., in Proceedings of the 9th International Particle Accelerator Conference (IPAC 2018), Vancouver (JACoW, Geneva, 2018), p. 2258.
[18] ASTRA manual, https://www.desy.de/ mpyflo/.

[19] L. Zheng et al., Phys. Rev. Accel. Beams 23, 052801 (2020).

[20] D. Dowell and J. Schmerge, Phys. Rev. Accel. Beams 12, 074201 (2009).

[21] R. Xiang et al., Phys. Rev. Accel. Beams 13, 043501 (2010).

[22] L. Xiao et al., in Proceedings of the 12th International Particle Accelerator Conference (IPAC2021), Campinas, Brazil (JACoW, Geneva, 2021).

[23] R. Huang, D. Filippetto, C. F. Papadopoulos, H. Qian, F. Sannibale, and M. Zolotorev, Phys. Rev. Accel. Beams 18, 013401 (2015).

[24] FLUKA manual; see http://www.fluka.org/fluka.php? id=manuals.

[25] M. Santana (private communication).

[26] GPT manual; see http://www.pulsar.nl/gpt/. 